\title{
New Fermi smearing approach for scattering of multi-GeV electrons by nuclei
}

\author{
A. Mariano* \\ Departamento de Física, Facultad de Ciencias Exactas, Universidad Nacional de la Plata, cc. 67, 1900 La Plata, Argentina \\ P. Podesta Lerma ${ }^{\dagger}$ \\ Departamento Física, Centro de Investigación y de Estudios Avanzados del IPN, Apartado Postal 14-740, 07000 México, D.F., Mexico
}

(Received 24 September 2003; published 16 March 2004)

\begin{abstract}
The cross section for electron scattering by nuclei at high momentum transfers is calculated within the Fermi smearing approximation, where binding effects on the struck nucleon are introduced via the relativistic Hartree approximation. The model naturally preserves current conservation, since the response tensor for an off-shell nucleon conserves the same form as for a free one but with an effective mass. Different parametrizations for the inelastic nucleon structure function are analyzed. The smearing at the Fermi surface is introduced through a momentum distribution obtained from a perturbative nuclear matter calculation. Recent CEBAF data on inclusive scattering of $4.05 \mathrm{GeV}$ electrons on ${ }^{56} \mathrm{Fe}$ are well reproduced for all measured geometries for the first time, as is evident from the comparison with previous calculations.
\end{abstract}

DOI: 10.1103/PhysRevC.69.034606

PACS number(s): 25.30.Dh, 11.80.-m, 89.75.Da

\section{INTRODUCTION}

Electron scattering by nuclei at high momentum transfers is a powerful tool to study the effective constituents of hadronic matter and exhibits very interesting new features. Within the $Q^{2} \equiv-q^{2}>1(\mathrm{GeV} / c)^{2}$ (or $q \equiv|\mathbf{q}|>1 \mathrm{GeV} / c$ ) domain, where $q \equiv(\omega, \mathbf{q})$ is the four-momentum transferred by the virtual photon, the struck nucleon is relativistic having momenta of the order of its mass $M$. In addition, for such regime the probability for exciting internal degrees of freedom of the nucleon (nucleon inelastic response) becomes increasingly important. Ideally, to describe the target response, one should start from a relativistic covariant theory of nuclei. However, such an approach is not practicable due to the difficulties in treating the meson exchange interactions. On the other hand, how to describe a nucleon with three momentum $\mathbf{p}$ inside the nucleus is a well known nonrelativistic nuclear structure problem. Thus, a model that couples both regimes is necessary. Electron scattering experiments have been described with a great variety of approximations, starting with the plane wave impulse approximation (PWIA). In the Born approximation the $A\left(e, e^{\prime}\right) A^{\prime}$ differential cross section reads

$$
\frac{d^{2} \sigma}{d \Omega^{\prime} d \epsilon^{\prime}}=\frac{e^{2}}{q^{4}} \frac{\mathrm{k}^{\prime}}{\mathrm{k}} L^{\mu \nu}\left(k, k^{\prime}\right) W_{\mu \nu}^{A}(\omega, \mathbf{q}),
$$

being $k, k^{\prime} \equiv|\mathbf{k}|,\left|\mathbf{k}^{\prime}\right|, \quad W_{\mu \nu}^{A}$ the nuclear response tensor, $L^{\mu \nu}\left(k, k^{\prime}\right)=1 / 2\left[k^{\prime \mu} k^{\nu}+k^{\mu} k^{\prime \nu}+\left(q^{2} / 2-m^{2} / 2\right) g^{\mu \nu}\right]$ the lepton tensor describing incoming and outgoing plane-wave electron states of four-momentum $k=\left(\epsilon \equiv \sqrt{\mathbf{k}^{\prime 2}+m^{2}}, \mathbf{k}\right)$ and $k^{\prime}$ $=\left(\epsilon^{\prime} \equiv \sqrt{\mathbf{k}^{\prime 2}+m^{2}}, \mathbf{k}^{\prime}\right)$, respectively, and $\Omega^{\prime} \equiv(\theta, \phi)$ the scattering angle. The PWIA lies on the following assumptions.

\footnotetext{
*Email address: mariano@venus.fisica.unlp.edu.ar

†Email address: podesta@fnal.gov
}

(i) The nuclear current operator can be written as the sum of the one-body nucleon currents.

(ii) The target decays virtually into an on-shell $(A-1)$ nucleus (spectator) and the off-shell $\left(p^{2} \neq M^{2}\right)$ struck nucleon, of four-momentum $p=\left(p_{0}, \mathbf{p}\right)$.

(iii) The nucleon that absorbs the photon is the same that leaves the target without interaction with the spectator, the final state interactions (FSI) being dropped. Under these suppositions, the nuclear response can be expressed as a convolution [1],

$$
W_{\mu \nu}^{A}(\omega, \mathbf{q})=\sum_{m_{t}} \int d E d \mathbf{p} P^{m_{t}}(E, \mathbf{p}) w_{\mu \nu}^{m_{t}}(p, q),
$$

of the nucleon response $w_{\mu \nu}^{m_{t}}(p, q)\left(m_{t}=1 / 2\right.$ and $-1 / 2$ for protons and neutrons, respectively) with the nuclear spectral function $P^{m_{t}}(E, \mathbf{p})$. This gives the joint probability of finding a nucleon with three-momentum $\mathbf{p}$ inside the target nucleus, and remove it with an energy $E=E_{B}+E_{A-1}^{\mathrm{exc}} . E_{B}=M_{A-1}+M$ $-M_{A}$ is the nucleon binding energy and $E_{A-1}^{\text {exc }}$ the excitation energy in which the residual nucleus is left. Notice that for an off-shell nucleon, the energy $p_{0}=p_{0}(E, \mathbf{p})$ depends on its removing energy and its three-momentum, thus to implement the PWIA or any extension including FSI one must address some important questions. First, the nucleon structure function is determined experimentally from proton or deuteron scattering on on-shell (free) targets, being $p^{2}=M^{2}\left(\right.$ or $\left.p_{0}=E_{\mathbf{p}} \equiv \sqrt{\mathbf{p}^{2}+M^{2}}\right)$. In our case we treat with an off-shell bounded nucleon with $p_{0} \neq E_{\mathbf{p}}$, and $p_{0}=p_{0}(E, \mathbf{p})$ depends on how the binding effects are included. Second, we need to extend the on-shell nucleon structure function to the off-shell regime to use it as input in the nuclear response calculation. The minimal hypothesis adopted in majority of works is to assume that $w_{\mu \nu}^{m_{t} \text { (off-shell) }}(p, q)$ $=w_{\mu \nu}^{m_{t}(\text { on-shell })}(\widetilde{p}, \widetilde{q})$, where $\widetilde{p}$ and $\widetilde{q}$ depend on the off-shell prescription adopted for $p_{0}=p_{0}(E, \mathbf{p})$. Third, whatever is the $(\widetilde{p}, \widetilde{q})$ pair we have a lack of the electromagnetic gauge 
invariance because $w_{\mu \nu}^{m_{t}(\text { off-shell })} q^{\nu} \neq 0$, due to the on-shell to off-shell extension. This brings in additional complications, a procedure required to restore current conservation $[1,2]$.

The old data coming from the NE3 SLAC experiment [3] were analyzed within different approaches. One of the first PWIA calculations included one-hole $(1 h)$ and two-particleone-hole $(2 p-1 h)$ excitations in $P^{m_{t}}(E, \mathbf{p})[1,4]$. The cross sections, when expressed in terms of the well known $x$ $\equiv Q^{2} /(2 M \omega)$ Bjorken variable, have been fairly well reproduced in the quasielastic peak ${ }^{1}(x \simeq 1)$ and inelastic $(x<1)$ regions, but underestimated for $x>1$. In order to correct this the FSI were introduced in different ways. For instance, when the PWIA has been extended by assuming a factorization hypothesis for the final nucleus wave function and by introducing pair correlations [5], the discrepancies in the region $1<x<2$ were circumvented. For $x>2$ more than two nucleons should be involved in the scattering process and thus the use of an optical potential was required [6]. Benhar et al. [7] have improved the PWIA results by introducing the FSI through an optical potential and by generating a folding function from the multiple-scattering Glauber theory and color transparency. The quite recent CEBAF $4.05 \mathrm{GeV}$ electron scattering [8] experiment covers the range $1<Q^{2}<7(\mathrm{GeV} / c)^{2}$ and $0.2 \lesssim x \leqq 4.2$, and vastly extends the angular and energy-loss range of the older NE3 SLAC one. Rinat and Taragin $[9,10]$ analyzed these results adopting an alternative approach to the PWIA. The nuclear response function is treated in a relativistic extension of the GerschRodriguez-Smith series [11], while the FSI were introduced through binary collisions. The CEBAF data are well reproduced for $x<1$ and in the left hand side neighborhood of the quasielastic peak $x \gtrsim 1$ [12]. Nevertheless, for all $\theta$ geometries the calculated cross section overestimates the data by a factor up to $2-10$ in the low-energy lost region $(x>1)$, being these discrepancies associated with defects in the adopted momentum distribution. In the present work we develop a modified version of the PWIA (in the sense that the nucleon behaves as free one but with an effective mass) where the off-shell effects and FSI are included via the relativistic Hartree approximation (RHA), being at the same time the gauge invariance preserved. The Fermi smearing effects are incorporated through a new nucleon momentum distribution, obtained from a perturbative calculation in nuclear matter. In addition, different parametrizations for the inelastic nucleon response measured at SLAC are analyzed. The CEBAF data are satisfactorily reproduced for all measured geometries, taking into account that the cross section varies over many orders of magnitude, the mentioned overestimation being avoided in the $x>1$ region.

\section{ELASTIC AND INELASTIC CROSS SECTIONS}

As the electron probes a region of dimensions $1 / q$, for high momentum transfers, surface effects are supposed to be

\footnotetext{
${ }^{1}$ The quasielastic peak energy for a nucleon at rest corresponds to $\omega_{q e} \equiv Q^{2} / 2 M$, which for $q / M \gg 1$ leads to $\omega_{q e} \simeq q$.
}

of minor importance and the nuclear matter framework is adopted. How good is this assumption will be analyzed in Sec. IV, where the theoretical results will be compared with the experimental and nuclear matter extrapolated data. The nucleon will be bounded by interaction with the scalar $\phi$ and vector $V_{\mu}$ mesons fields, within the framework of quantum hadrodynamics $[13,14]$. The nucleus response tensor is the Lorentz invariant amplitude and reads [15]

$$
\begin{aligned}
W_{\mu \nu}^{A}(\omega, \mathbf{q})= & \frac{\mathrm{k} M_{A}}{\sqrt{\left(k \cdot P_{A}\right)^{2}-m^{2} M_{A}^{2}}} \frac{V}{(2 \pi)^{3}} \\
& \times \sum_{\mathbf{p}^{\prime} m_{s}^{\prime} m_{t}^{\prime}} \sum_{f}\left\langle P_{A}\left|\hat{J}(0)_{\mu}\right| p^{\prime} m_{s}^{\prime} m_{t}^{\prime}, P_{A-1}^{f}\right\rangle \\
& \times\left\langle p^{\prime} m_{S}^{\prime} m_{t}^{\prime}, P_{A-1}^{f}\left|\hat{J}(0)_{\nu}\right| P_{A}\right\rangle \\
& \times(2 \pi)^{4} \delta\left(P_{A}+k-k^{\prime}-P_{A-1}^{f}-p^{\prime}\right),
\end{aligned}
$$

being $P_{A}=\left(M_{A}, 0\right)$ and $P_{A-1}^{f}=\left[\sqrt{\mathbf{p}_{f}^{2}+\left(M_{A-1}^{f}\right)^{2}}, \mathbf{p}_{f}\right]$ the target and residual nucleus four-momentum, respectively, with the mass $M_{A-1}^{f}=M_{A-1}+\omega_{A-1}^{f}$ including the excitation energy $\omega_{A-1}^{f}$. The sum on $f$ encloses the set of final residual nucleus states. We also sum on the final states of the struck nucleon with four-momentum $p^{\prime}=\left(p_{0}^{\prime}, \mathbf{p}^{\prime}\right)$, spin $m_{s}^{\prime}$ and isospin $m_{t}^{\prime}$, with density $V /(2 \pi)^{3}$ in the quantization volume $V . \hat{J}(x)$ is the effective hadron current density operator $\quad \hat{J}_{\mu}(x)=i \bar{\psi}(x) \Gamma_{\mu}(q) \psi(x) \quad$ with $\quad \Gamma_{\mu}(q)=F_{1}\left(q^{2}\right) \gamma_{\mu}$ $+i F_{2}\left(q^{2}\right) \kappa / 2 M \sigma_{\mu \nu} q^{\nu}$ for the nucleon elastic response case, being $\psi(x)$ and $\kappa$ the nucleon field and anomalous magnetic moment, respectively.

We are going to develop on the same footing the nuclear response calculation within the mean field theory (MFT) (where the meson fields are approximated by their vacuum spectation, i.e., constant, values), and in the RHA [13] (where vacuum fluctuation corrections are added to the MFT results). Later, when we compare the calculated cross section with the data, the RHA election will be justified. The nucleon field is expanded as

$$
\begin{aligned}
\psi(x)= & \frac{1}{\sqrt{V}} \sum_{\mathbf{p} m_{s} m_{t}} \sqrt{\frac{M^{*}}{E_{\mathbf{p}}^{*}}}\left[u\left(\mathbf{p} m_{s} m_{t}\right) a_{\mathbf{p} m_{s} m_{t}} e^{i p x}\right. \\
& \left.+b_{\mathbf{p} m_{s} m_{t}}^{\dagger} v\left(\mathbf{p} m_{s} m_{t}\right) e^{-i p x}\right],
\end{aligned}
$$

where the single particle spectrum is given by

$$
p_{0}=C_{V}^{2} \frac{\rho_{B}}{M^{2}}+E_{\mathbf{p}}^{*}
$$

with $E_{\mathbf{p}}^{*}=\sqrt{\mathbf{p}^{2}+M^{* 2}}$ and $M^{*} \equiv M+\Sigma\left(C_{S}, M^{*}\right) . M^{*}<M$ is the effective mass acquired by the nucleon by action of the attractive scalar field and is determined self-consistently [13] through the scalar self-energy $\Sigma \equiv \Sigma_{M F T}$ or $\Sigma_{R H A} . \Sigma_{M F T}$ includes the tadpole diagram (a) in Fig. 1, retaining in its evaluation only the contribution from nucleons in the filled Fermi sea in the nucleon propagator (thick full lines). $\Sigma_{R H A}$ includes the same diagram but the full nucleon propagator (which encloses the contribution of the occupied negative-energy states) is used in the 


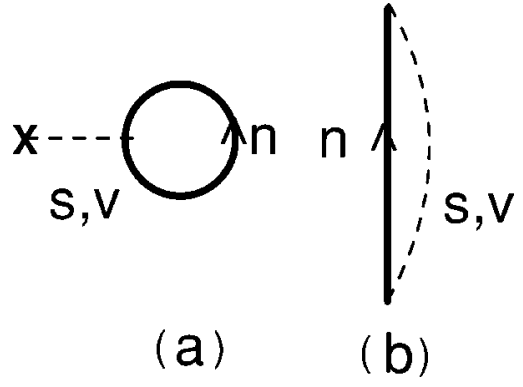

FIG. 1. (a) Tadpole diagram included in the MFT and RHA self-energies. (b) Tadpole exchange diagram that is added in order to get the relativistic Hartree-Fock self-energy. The dashed lines indicate the propagator of the scalar $(S)$ or vector meson $(V)$ that interacts with a nucleon $n$ (full lines).

evaluation of the self-energy. Then the MFT or the RHA are derived by summing up the self-energy to all orders through the self-consistent determination of $M^{*}$, being this procedure convergent in both cases. The first term in Eq. (5) accounts for the action of the repulsive vector field. $C_{V}$ and $C_{S}$ are the two free parameters [16], which depend on the meson coupling constants and masses, fixed to reproduce the experimental binding energy per nucleon of $-16 \mathrm{MeV}$ at the Fermi momentum $p_{F}=1.42 \mathrm{fm}^{-1}$ (or the baryon density $\rho_{B}=0.19 \mathrm{fm}^{-3}$ ) for the normal nuclear matter. Assuming that the residual nucleus is left in its ground state and adopting the prescriptions (ii) and (iii) mentioned above, the response tensor can be obtained from Eqs. (3)-(5) as

$$
W_{\mu \nu}^{A}(q)=2 \sum_{m_{t}} \int d \mathbf{p} \frac{M^{*}}{E_{\mathbf{p}}^{*}} n^{m_{t}}(\mathbf{p}) w_{\mu \nu}^{m_{t}}\left(p^{*}, q\right),
$$

where the factor 2 resembles the sum over spin states, and

$$
\begin{aligned}
w_{\mu \nu}^{m_{t}}\left(p^{*}, q\right)= & w_{e 1}^{m_{t}}\left(Q^{2}, \nu^{*}\right)\left[-g_{\mu \nu}+\frac{q_{\mu} q_{\nu}}{q^{2}}\right]+w_{e 2}^{m_{t}}\left(Q^{2}, \nu^{*}\right) \\
& \times\left[\frac{p_{\mu}^{*}}{M^{*}}-\nu^{*} \frac{q_{\mu}}{q^{2}}\right]\left[\frac{p_{\nu}^{*}}{M^{*}}-\nu^{*} \frac{q_{\nu}}{q^{2}}\right]
\end{aligned}
$$

with $p^{*}=\left(E_{\mathbf{p}}^{*}, \mathbf{p}\right)$ and ${ }^{2} \nu^{*}=p^{*} \cdot q / M^{*} \cdot n^{m_{t}}(\mathbf{p})$ is the nucleon momentum distribution in the target ground state $\left|0_{A}\right\rangle$,

$$
n^{m_{t}}(\mathbf{p})=\frac{V}{(2 \pi)^{3}}\left\langle 0_{A}\left|a_{\mathbf{p} m_{s} m_{t}}^{\dagger} a_{\mathbf{p} m_{s} m_{t}}\right| 0_{A}\right\rangle
$$

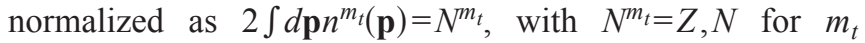
$=1 / 2,-1 / 2$. The elastic Lorentz scalar functions present in Eq. (7) are

$$
w_{e 1}^{m_{t}}\left(Q^{2}, \nu^{*}\right)=\tau G_{M}^{m_{t} 2}\left(Q^{2}\right) \delta\left(\nu^{*}-\frac{Q^{2}}{2 M^{*}}\right),
$$

$\overline{{ }^{2} Q^{2} \text { and }} \nu=p . q / M$ are commonly used as independent variables for $w_{1,2}^{m_{t}}$ in the nucleon response.

$$
w_{e 2}^{m_{t}}\left(Q^{2}, \nu^{*}\right)=\frac{G_{E}^{m_{t}{ }^{2}}\left(Q^{2}\right)+\tau G_{M}^{m_{t}{ }^{2}}\left(Q^{2}\right)}{1+\tau} \delta\left(\nu^{*}-\frac{Q^{2}}{2 M^{*}}\right),
$$

where $\quad G_{E}^{m_{t}}\left(Q^{2}\right)=F_{1}^{m_{t}}\left(Q^{2}\right)-F_{2}^{m_{t}}\left(Q^{2}\right) \kappa^{m_{t}} \tau \quad$ and $\quad G_{M}^{m_{t}}\left(Q^{2}\right)$ $=F_{1}^{m_{t}}\left(Q^{2}\right)+F_{2}^{m_{t}}\left(Q^{2}\right) \kappa^{m_{t}}$ are the electric and magnetic form factors, and $\tau=Q^{2} / 4 M^{* 2}$. In the numerical calculations we adopt the Sachs form for them, assuming that they do not change in the nuclear medium [17]. Equations (7), (9), and (10) show that the MFT or RHA lead to the prescription $w_{\mu \nu}^{m_{t} \text { (off-shell) }}(p, q)=w_{\mu \nu}^{m_{t} \text { (on-shell) }}\left(p^{*}, q\right)$ and $w_{e 1,2}^{\text {(off-shell) }}\left(Q^{2}, \nu\right)$ $=w_{e 1,2}^{\text {(on-shell) }}\left(Q^{2}, \nu^{*}\right)$, for the elastic case. The nucleon spinors carry a four-momentum $p^{*}$ being $p^{* 2}=M^{* 2}$, and as $M^{*}<M$ this makes us remember that the struck nucleon is bounded. Lorentz, parity, and gauge invariances are now also fulfilled as were for a nucleon of mass $M$, as a consequence of the form of Eq. (7) [18]. FSI are included since the nucleon is bounded also after the interaction with the photon. For $Q^{2}>1(\mathrm{GeV} / c)^{2}$ the probability of exciting internal states of the nucleon is important, and a replacement $w_{e 1,2}^{m_{t}} \rightarrow w_{1,2}^{m_{t}}=w_{e 1,2}^{m_{t}}+w_{i 1,2}^{m_{t}}$ in Eq. (7) should be done, adding an inelastic contribution $w_{i 1,2}^{m_{t}}$. For $w_{i 1,2}^{m_{t}}$ we use different parametric fits done at SLAC for $p\left(e, e^{\prime}\right) p^{\prime}$ and $d\left(e, e^{\prime}\right) d^{\prime}$ data through Eqs. (7), with $M^{*}=M$. We assume that the recipe $w_{i 1,2}^{\text {off-shell }}\left(Q^{2}, \nu\right)=w_{i 1,2}^{\text {on-shell }}\left(Q^{2}, \nu^{*}\right)$, which naturally appears in the elastic case, is also valid for the inelastic nucleon response function. Finally, the decomposition $w_{1,2}^{m_{t}}=w_{e 1,2}^{m_{t}}+w_{i 1,2}^{m_{t}}$ leads also to split the inclusive cross section (1) in elastic and inelastic contributions.

\section{NUCLEON MOMENTUM DISTRIBUTION}

From Eqs. (2) and (6) it is clear that we are working within the Fermi smearing approximation (FSA) $P^{m_{t}}(E, \mathbf{p})$ $\sim n^{m_{t}}(\mathbf{p}) \delta\left(E-E_{B}\right)$, giving $n^{m_{t}}(\mathbf{p})$ the probability of finding a nucleon with momentum $\mathbf{p}$, and isospin $m_{t}$ in the target $\left|0_{A}\right\rangle$. $n^{m_{t}}(\mathbf{p})$ is calculated in a $0 p 0 h+2 p 2 h+4 p 4 h$ configuration space for the $A$ target, being

$$
\begin{aligned}
& \left|0_{A}\right\rangle=\mathcal{N}\left[|0 p 0 h\rangle+\frac{1}{(2 !)^{2}} \sum_{p^{\prime} s, h^{\prime} s} c_{p_{1} p_{2} h_{1} h_{2}}\left|p_{1} p_{2} h_{1} h_{2}\right\rangle\right. \\
& \left.+\frac{1}{(4 !)^{2}} \sum_{p^{\prime} s, h^{\prime} s} c_{p_{1} p_{2} p_{3} p_{4} h_{1} h_{2} h_{3} h_{4}}\left|p_{1} p_{2} p_{3} p_{4} h_{1} h_{2} h_{3} h_{4}\right\rangle\right] \text {, }
\end{aligned}
$$

where these $|n p n h\rangle$ (with $n=0,2,4)$ stand for the unperturbed states. In this way in the residual nucleus we have $1 h$, $2 p 3 h, 4 p 5 h, 1 p 2 h$, and $3 p 4 h$ excitations when the struck nucleon is removed. The residual nucleon-nucleon interaction is included within a perturbative approach as in Ref. [19] by expanding the coefficients $c_{2 p 2 h}$ and $c_{4 p 4 h}$ up to the first and second order, respectively. This "minimum" perturbative scheme allows to include norm corrections $\mathcal{N}$ $=\left\langle 0_{A} \mid 0_{A}\right\rangle^{-1}$, avoiding in this way contributions of unbalanced disconnected diagrams. We get 


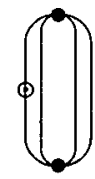

(a)

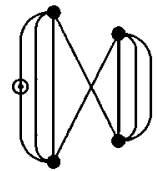

(b)

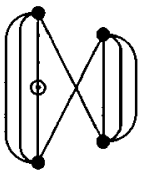

(c)

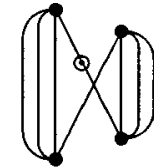

(d)

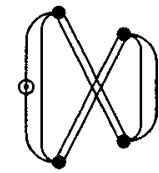

(e)
FIG. 2. Goldstone diagrams corresponding to the second-order $2 p 2 h$ correction $\delta n^{(2)}(p)$ (a) and fourth-order $4 p 4 h$ correction $\delta n^{(4 C)}(p)(\mathrm{b}),(\mathrm{c}),(\mathrm{d}),(\mathrm{e})$. Each line indicates schematically a particle or a hole state, the dots represent the residual interaction, and the encircled dots correspond to the number operator $n(\mathbf{p})$. With (b), (c), (d), and (e) we indicate different ways to attach the number operator to a particle or hole line in $\delta n^{(4 C)}(\mathbf{p})$.

$$
n^{m_{t}}(\mathbf{p})=\frac{3 N^{m_{t}}}{4 \pi p_{F}^{3}}\left[\theta(1-p)+\delta n^{(2)}(p)+\delta n^{(4 C)}(\mathrm{p})\right],
$$

where $\mathbf{p} \equiv|\mathbf{p}|$ is measured in units of the Fermi momentum $p_{F}$. The first term is the usual $0 p 0 h$ Fermi step function, while $\delta n^{(2)}(p)$ and $\delta n^{(4 C)}(p)$ (where the superscript $C$ indicates "connected" $4 p 4 h$ diagrams) enclose $2 p 2 h$ and $4 p 4 h$ contributions, respectively, which deplete it. The expressions for $\delta n^{(2)}(p)$ and $\delta n^{(4 C)}(p)$, are given in Ref. [19], while the Goldstone diagrams corresponding to them are shown in Fig. 2.

\section{RESULTS AND CONCLUSIONS}

We now compare the differential cross section calculated within our model, with the CEBAF experimental results for ${ }^{56} \mathrm{Fe}[8]$ for the various accessible geometries $\theta$ $=15^{\circ}, 23^{\circ}, 30^{\circ}, 37^{\circ}, 45^{\circ}, 55^{\circ}, 74^{\circ}$. One of the parametrizations for $w_{i 1,2}^{m_{t}}$ was found by Bodek et al. [4] in the kinematical range $1<Q^{2}<20(\mathrm{GeV} / c)^{2}$ and $0.1 \leqslant x \leqslant 0.77$. The other one was reported by Whitlow [20], and corresponds to the range $0.6<Q^{2}<30(\mathrm{GeV} / c)^{2}$ and $0.06 \leqslant x \leqslant 0.9$.

The functions obtained in these parametrizations are described in detail in Ref. [14], and as they do not cover all the low-energy and momentum transfer region of CEBAF, an extrapolation is necessary. This fact could introduce some uncertainties in the calculation. Within the MFT and for ${ }^{56} \mathrm{Fe}$ $\left(p_{F}=1.36 \mathrm{fm}^{-1}\right), M^{*}=0.648 M$. This value is too low to reproduce satisfactorily the total cross section since the quasielatic peak is shifted too much to the right and its width $\left(\Delta \omega_{q e}\right)$ is enlarged in excess, as shown in Fig. 3. This behavior of the MFT at high momentum transfers was analyzed in Ref. [2] for the longitudinal response, where a dependence of

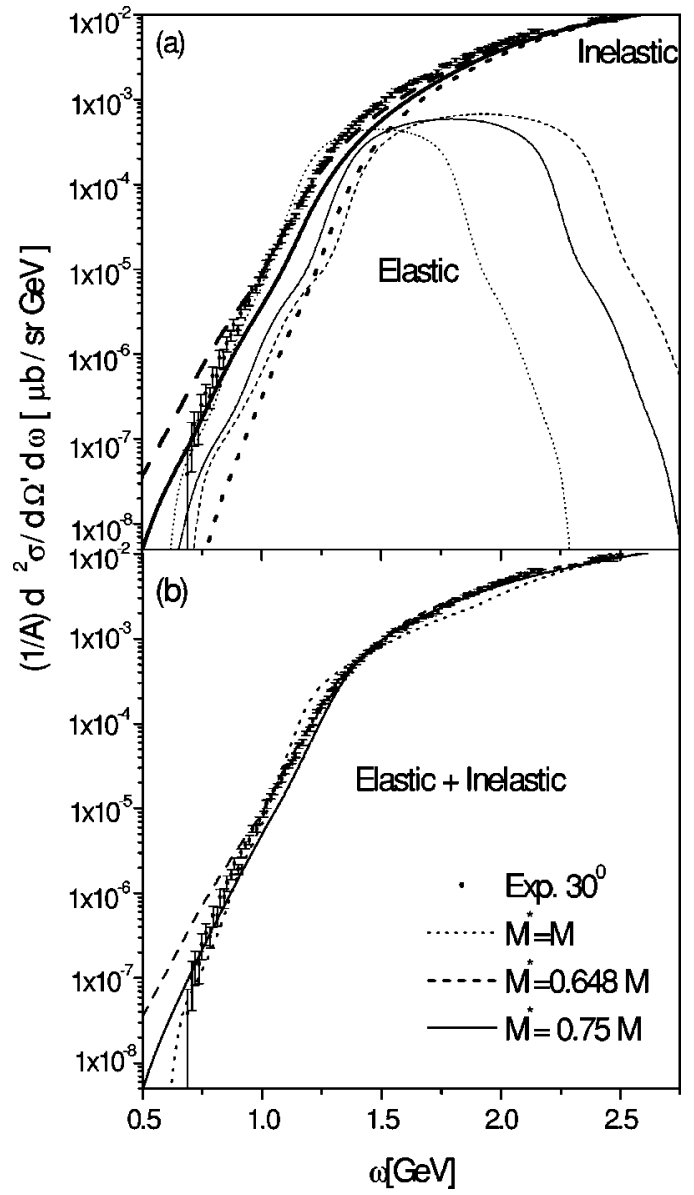

FIG. 3. We show the sensibility with the effective mass $M^{*}$ of the quasielastic and inelastic contributions to the cross sections per nucleon for ${ }^{56} \mathrm{Fe}$. Here the replacement $\epsilon^{\prime}=\epsilon+\omega$ is done. In the panel (a) both cross sections are shown separately for the values $M^{*}=1,0.64$, and 0.74 . Thin lines indicate elastic cross sections while thick lines indicate the inelastic one. In panel (b) the total elastic+inelastic cross section is shown for the different values of $M^{*}$. Again, experimental results come from Ref. [8].

$\phi$ and $V_{\mu}$ with $\mathrm{p}$ was introduced. ${ }^{3}$ The value of $\omega_{q e}$ is certainly corrected (but not the detailed peak's shape) since as it is shown, the binding-energy shift effects are diminished as $p$ increases. Nevertheless the $\mathrm{p}$ dependence of the fields carries a gauge invariance violation that is corrected by introducing a (nonunique) vertex correction in $\Gamma_{\mu}$. The Eqs. (7), (9), and (10) are altered, and the prescription $w_{e 1,2}^{\text {off-shell }}\left(Q^{2}, \nu\right)$ $=w_{e 1,2}^{\text {on-shell }}\left(Q^{2}, \nu^{*}\right)$ is no longer valid. This brings a problem at the moment of introducing the inelastic response, since the assumption $w_{i 1,2}^{\text {off-shell }}\left(Q^{2}, \nu\right)=w_{i 1,2}^{\text {on-shell }}\left(Q^{2}, \nu^{*}\right)$ is not justified. Alternatively, we try to improve the MFT description by adding the vacuum fluctuation corrections to $\Sigma_{M F T}$ [13], and go to the RHA [22] where $M^{*}=0.74 M$. Equations (7), (9), and (10) are now still valid. As can be seen in Fig. 3, for RHA the binding-energy shift is more moderated and the width is di-

\footnotetext{
${ }^{3}$ Within the Hartree-Fock approach this dependence is generated naturally by adding in the self-energy the exchange graph (b) of Fig. 1, and summing up to all orders.
} 


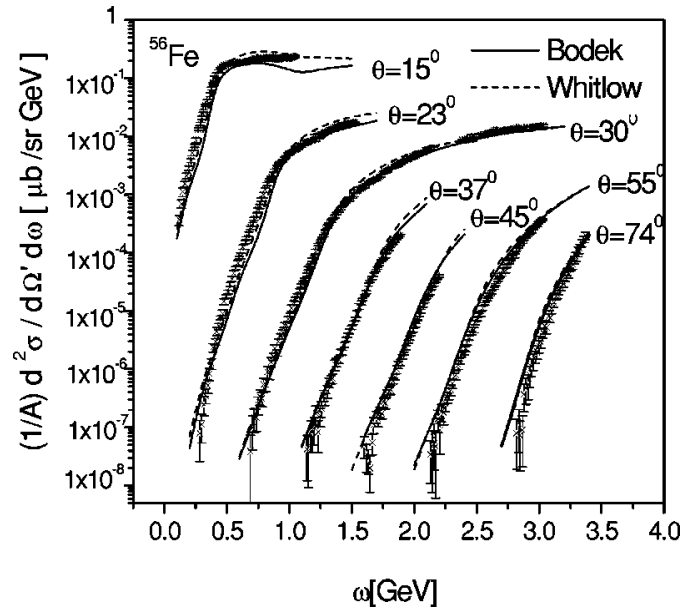

FIG. 4. Calculated differential cross section per nucleon for different $\theta$ geometries for ${ }^{56} \mathrm{Fe}$. Experimental data come from Ref. [8]. Results are shown for both, the fitting of Bodek and Withlow of the inelastic nucleon response, and for a value $M^{*}=0.74$ corresponding to the RHA.

minished, getting a better description for the total cross section. This improvement is not casual since as is well known the RHA yields to the "best" single-particle spectrum in the sense that it minimizes the energy of the whole system. In addition when the longitudinal response for electron scattering at $q=0.55 \mathrm{GeV}$ and $1.14 \mathrm{GeV}$ transfers is analyzed, we get values $\omega_{q e}=0.182 \mathrm{GeV} \quad\left(\Delta \omega_{q e}=0.302 \mathrm{GeV}\right)$ and $0.615 \mathrm{GeV}(0.436 \mathrm{GeV})$, respectively, in full agreement with the results reported in Ref. [2]. This indicates that keeping $p$-independent fields and changing the value of $M^{*}$ in Eqs. (9) and (10), one can still improve the quasielastic peak position both at low and high momentum transfers at the same time. FSI are taken into account in our model at the RHA level. Binding effects are present in the final state, since the nucleon still has mass $M^{*}$ after absorbing the photon. This simple form of introducing FSI has never been used previously to describe a multi-GeV electron experiment with the inclusion of the inelastic nucleon response, being only described in the past the quasielastic cross section at intermediate energies in the MFT framework [23].

FSI affect directly the quasielastic response defined in Eqs. (7), (9), and (10), since the size and position of the quasielastic peak are controlled by $\tau=Q^{2} / 4 M^{* 2}$ (which scales quadratically with $M^{*}$ ) and $\nu^{*}$, respectively. In the inelastic nucleon response, FSI effects are included indirectly through the replacement $\nu \rightarrow \nu^{*}=p^{*} q / M^{*}$ in the $w_{i 1,2}^{m_{t}}$ on-shell functions. Our results for the total cross section are shown in Fig. 4. As can be seen, the overall agreement is good for all angles $\theta$, considering that the cross section varies over several decades. At $\omega<\omega_{q e}(x>1)$ Withlow's fit seems to be preferred to Bodek's, which is due possibly to differences in the extrapolation for the $x>1$ range. For $\omega>\omega_{q e}(x<1)$ the behavior is opposite. We see that the model tends to overestimate the $x>1$ data, in the last two $\theta$ values. The inelastic response dominates the cross section at these geometries since $Q^{2} \gtrsim 4(\mathrm{GeV} / c)^{2}$, and this overestimation could be also as a consequence of uncertainties in the extrapolation for

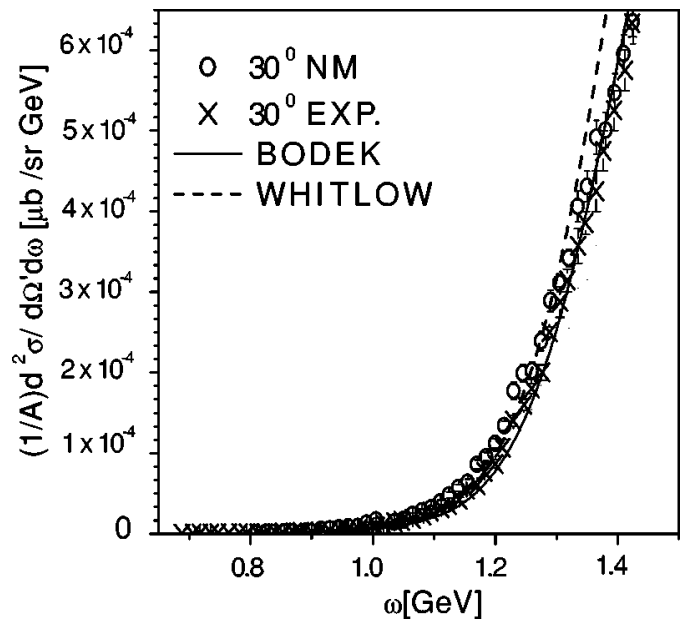

FIG. 5. Comparison of the extrapolated nuclear matter (NM) cross section per nucleon at $\epsilon=4 \mathrm{GeV}$ reported in Ref. [3], with the experimental data at $\epsilon=4.05 \mathrm{GeV}$ [8] and our calculations, for the indicated geometry.

$x>1$. We conclude that to implement the generated nuclear matter momentum distribution within the RHA framework is a consistent approach for treating electron scattering at these momentum transfers. This is further supported by the following observations.

(i) In Fig. 5 we compare the nuclear matter extrapolated cross section reported in Ref. [3] for $\epsilon=4 \mathrm{GeV}$ and $\theta=30^{\circ}$ in the shown energy lost region, with the experimental results of Ref. [8] and our calculations. As can be seen, the extrapolated results for this geometry are larger than the experimental data, being the difference small comparatively to the range of variation of the cross section (in Fig. 5 we show only an interval of the full energy lost region). This indicates that nuclear matter is a reasonable framework at these momentum transfers. Our nuclear matter results change from below the data to above the extrapolation, indicating that some improvements as would be a momentum dependence of the effective mass or higher-order contributions to the momentum distribution should be more deeply analyzed.

(ii) We are working within the FSA that in previous calculations with other nuclear matter momentum distributions $[24,25]$ lead to a large overestimation of the cross section at low electron energy loss $[1,26]$. As can be seen from Eq. (12) and Fig. 2, we are including fourth-order corrections to $n(\mathbf{p})$ in addition to the usual second-order contributions [24]. In Ref. [19], it has been shown that the second-order perturbation approach overestimates the depletion of the Fermi surface and thus the high momentum tail of the momentum distribution. This could be the reason of the mentioned overestimation present in previous nuclear matter calculations. In Fig. 6 we show the momentum distribution obtained from Eq. (12) together with its second-order approach, being $\delta n^{(4 C)}(\mathrm{p})$ dropped. In the same figure we show the momentum distribution of Ref. [24] (parametrized in Ref. [6]), which was obtained within a second-order perturbation approach over a set of unperturbed variational wave functions.

It is important to mention that FSI also are responsible for the behavior of the cross section in the low-energy lost re- 


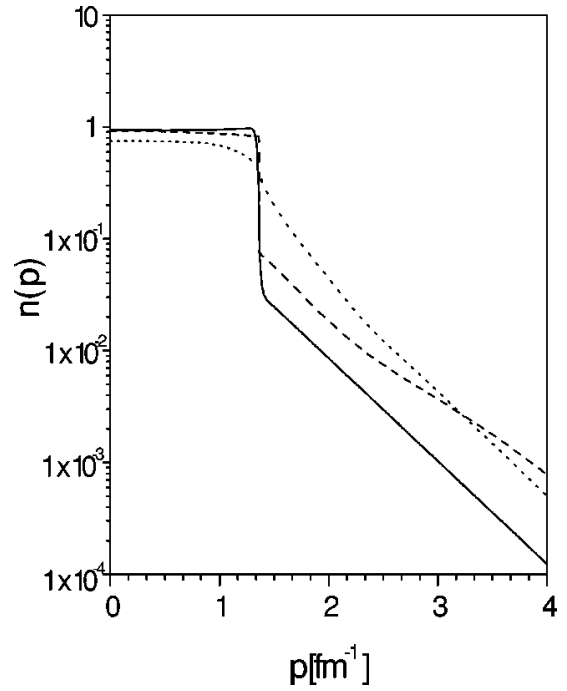

FIG. 6. Comparison of the nuclear matter momentum distribution used in our calculations (full lines), with its second order approach (dotted lines) and the momentum distribution of Ref. [24] (dashed lines).

gion. In our model FSI are taken into account by using an effective nucleon mass, and different values of $M^{*}$ lead to different contributions to the cross section in the mentioned region, as can be seen from Fig. 3. The value of $M^{*}$ $=0.74 M$ within the RHA seems to introduce FSI consistently with the implemented momentum distribution.

(iii) Finally the overestimation by a factor $2-10$ in previous theoretical evaluations of the cross section at $x>1$ [12] is not present in our calculation. This can be seen in Fig. 7 where we compare the ratios of the theoretical to experimental cross section, for some selected geometries where the differences are appreciable, in our model and in that of Ref. [12].

In summary, to treat the scattering of $\mathrm{GeV}$ electrons by nuclei we have implemented a new Fermi smearing approach. Binding effects and FSI are introduced through the nucleon effective mass within the RHA, which leads to better results than the plain MFT [14]. In the model, current conservation is preserved naturally without ad hoc modifications in the structure functions. Fermi smearing effects are introduced through a new momentum distribution that accounts for $2 p 2 h$ and $4 p 4 h$ correlations in the target, generated via a perturbative approach in nuclear matter. We get a reasonable overall description of the behavior of the measured cross section at CEBAF, for the scattering of $4.05 \mathrm{GeV}$ electrons on ${ }^{56} \mathrm{Fe}$. The agreement for all the accessible geometries has

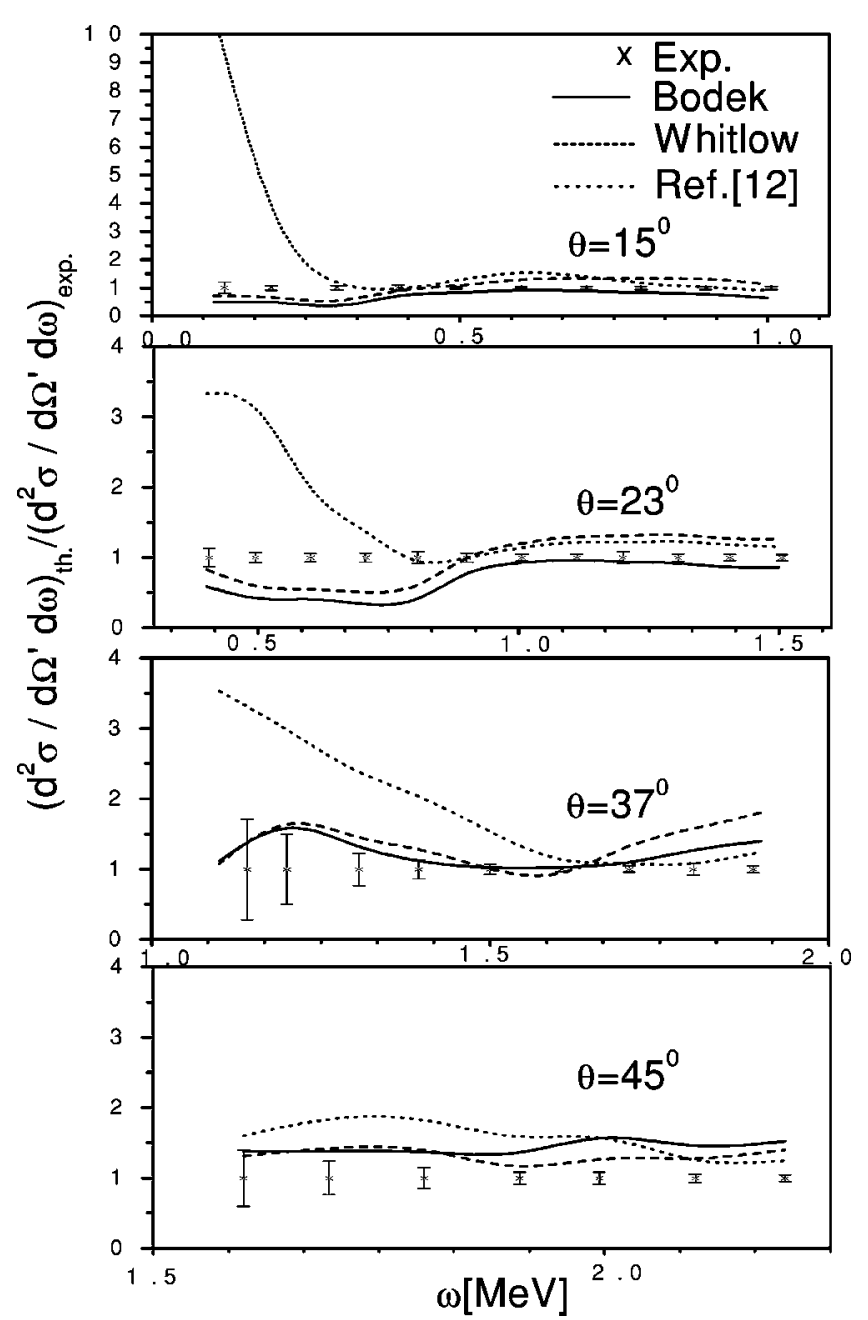

FIG. 7. Ratio of the theoretical to experimental cross section for some selected geometries, within our model (full and dashed lines) and the model of Ref. [12] (dotted lines).

been significantly improved in comparison with previous theoretical studies [12]. It could also suggest that within the model the FSI and Fermi smearing effects combine consistently, this could be more clearly established examining the effect of changing $M^{*}$ on the contributions to the cross section coming from the hole and particle strength functions. This more detailed analysis and the scaling behavior of the model will be reported elsewhere [21].

\section{ACKNOWLEDGMENT}

The Work of A. M. was partially supported by Conicet (Argentina).
[1] C. Ciofi degli Atti and S. Simula, Phys. Rev. C 43, 1155 (1991); C. Ciofi degli Atti, D. B. Day, and S. Liuti, ibid. 46, 1045 (1992).

[2] M. R. Frank, Phys. Rev. C 49, 555 (1994); Hungchong Kim, C. J. Horowitz, and M. R. Frank, ibid. 51, 792 (1994).

[3] D. B. Day et al., Phys. Rev. Lett. 59, 427 (1987); D. B. Day et al., Phys. Rev. C 40, 1011 (1989); D. B. Day et al., ibid. 48, 1849 (1993).

[4] A. Bodek and J. L. Ritchie, Phys. Rev. D 23, 1070 (1981).

[5] C. Ciofi degli Atti and S. Simula, Phys. Lett. B 325, 276 (1994).

[6] C. Ciofi degli Atti and S. Simula, Phys. Rev. C 53, 1689 
(1996)

[7] O. Benhar, A. Fabrocini, S. Fantoni, G. A. Miller, V. R. Pandharipande, and I. Sick, Phys. Rev. C 44, 2328 (1991).

[8] J. R. Arrington et al., Phys. Rev. Lett. 82, 2056 (1999).

[9] S. A. Gurvitz and A. S. Rinat, Prog. Part. Nucl. Phys. 34, 235 (1995).

[10] A. S. Rinat and M. F. Taragin, Nucl. Phys. A620, 417 (1997).

[11] H. A. Gersch, L. J. Rodriguez, and Phil N. Smith, Phys. Rev. A 5, 1547 (1973).

[12] A. S. Rinat and M. F. Taragin, Phys. Rev. C 60, 044601 (1999); A. S. Rinat, M. F. Taragin, and M. Viviani, nucl-th/ 0307004.

[13] B. D. Serot and J. D. Walecka, Adv. Nucl. Phys. 16, 1 (1986).

[14] T. C. Ferrée and D. S. Koltun, Phys. Rev. C 55, 253 (1997).

[15] J. D. Bjorken and S. D. Drell, Relativistic Quantum Mechanics (McGraw-Hill, New York, 1964).

[16] J. D. Walecka, Theoretical Nuclear and Subnuclear Physics
(Oxford University Press, New York, 1995), Chap. 39.

[17] D. S. Koltun and T. S. Ferreé, Phys. Rev. C 52, 901 (1995).

[18] S. D. Drell and J. D. Walecka, Ann. Phys. (N.Y.) 28, 18 (1964).

[19] A. Mariano, F. Krmpotic, and AFR. de Toledo Piza, Phys. Rev. C 53, 1664 (1996).

[20] L. W. Whitlow, SLAC Report No. 357, 1990.

[21] A. Mariano et al. (unpublished).

[22] S. A. Chin, Ann. Phys. (N.Y.) 108, 301 (1977).

[23] R. Rosenfelder, Ann. Phys. (N.Y.) 128, 188 (1980).

[24] S. Fantoni and V. R. Pandharipande, Nucl. Phys. A427, 473 (1984).

[25] O. Benhar, A. Fabrocini, and S. Fantoni, Nucl. Phys. A505, 267 (1989).

[26] O. Benhar, A. Fabrocini, S. Fantoni, and I. Sick, Nucl. Phys. A579, 493 (1994). 